\title{
Erratum to: Lessons learned from comparing molecular dynamics engines on the SAMPL5 dataset
}

\author{
Michael R. Shirts ${ }^{1} \cdot$ Christoph Klein $^{2} \cdot$ Jason M. Swails $^{3} \cdot$ Jian Yin $^{4}$. \\ Michael K. Gilson ${ }^{4}$ David L. Mobley ${ }^{5}$ David A. Case ${ }^{3} \cdot$ Ellen D. Zhong $^{6}$
}

Published online: 27 July 2017

(C) Springer International Publishing AG 2017

\section{Erratum to: J Comput Aided Mol Des}

(2017) 31:147-161

DOI 10.1007/s10822-016-9977-1

Electronic Supplementary Material (ESM) (referred to in the article as Supporting Information) had erroneously not been published in the original publication. The missing ESM are provided in this erratum.

Electronic supplementary material The online version of this article contains supplementary material (doi:10.1007/s10822017-0043-4), which is available to authorized users.

The online version of the original article can be found under doi:10.1007/s10822-016-9977-1.

\section{Michael R. Shirts}

michael.shirts@ colorado.edu

1 Department of Chemical and Biological Engineering, University of Colorado Boulder, Boulder, CO, USA

2 Department of Chemical Engineering, Vanderbilt University, Nashville, TN, USA

3 Department of Chemistry and Chemical Biology, Rutgers University, New Brunswick, NJ, USA

4 Skaggs School of Pharmacy and Pharmaceutical Sciences, University of California San Diego, La Jolla, CA, USA

5 Departments of Pharmaceutical Sciences and Chemistry, University of California, Irvine, Irvine, CA, USA

6 Department of Chemical Engineering, University of Virginia, Charlottesville, VA, USA 